\title{
Coherent choice and epistemic entrenchment (Preliminary report)
}

\author{
Hans Rott \\ University of Konstanz, Department of Philosophy \\ P.O. Box 5560, 78434 Konstanz, Germany \\ pirott@nyx.uni-konstanz.de
}

\begin{abstract}
Belief revision has recently been modelled with the help of the notion of epistemic entrenchment (Gärdenfors and Makinson, TARK 1988 , and Rott, JoLLI 1992). This paper offers a systematic justification of the postulates for entrenchment by interpreting entrenchment in terms of the general theory of rational choice. A translation is specified from conditions for entrenchment into conditions for choice, and vice versa. The relative strengths of the postulates are studied, and connections with related work are discussed.
\end{abstract}

\section{Introduction}

The notion of the epistemic entrenchment has recently attracted quite some attention in the AI community. It was introduced, first under the name "epistemic importance", by Peter Gärdenfors [7, 8]. He specified two possible "origins" of epistemic entrenchment: an information-theoretic appoach and a paradigm approach. However, neither of these approaches squares well with the logical constraints which are placed on entrenchment relations in the later and more mature work of Gärdenfors and Makinson [10]. Although it is now known how epistemic entrenchment can be put to work technically, the problem of providing a systematic justification of the postulates for entrenchment relations is still largely unresolved. The present paper offers a solution to this problem which is faithful to the literal meaning of the term "entrenchment".

We follow the philosophical strategy of reducing logical principles to abstract principles of rationality for decisions and actions which we consider to be more fundamental than the former. The standard theory of epistemic entrenchment $[10,21]$ is interpreted in terms of the general theory of rational choice, and the following questions are being addressed. Are the usual postulates for entrenchment justified by principles of rational choice? Do the latter principles, or the mere interpretation of entrenchments in terms of choice, impose interesting additional constraints on entrenchment relations? Conversely, which principles of rational choice can be retrieved from the entrenchment postulates, and what is their status in the theory of choice? 


\section{Basic Principles}

The following considerations presuppose a propositional language that contains at least the binary connective ' $\wedge$ ' for conjunction. No other connectives will be needed for our discussion. An epistemic state, or a knowledge base, will be represented by the set $K$ of sentences which are believed or accepted by an agent in that epistemic state (in the knowledge base). Revision and contraction functions are used for the representation of changes of belief. The general format is this: (old knowledge base, set to retract) $\longmapsto$ new knowledge base. The retract set $S$ consists of the set of sentences at least one of which has to be given up. The pick contraction of $K$ with respect to $S$, denoted $K \dot{-}\langle S\rangle$, is the minimal (most economic) contraction of the belief set $K$ that is necessary in order to discard at least one of element of $S$. It is left to the agent's decision which element(s) of $S$ is (are) best to give up.

We assume in this paper that the beliefs of an agent are closed under conjunction:

$$
\left\{\phi_{1}, \ldots, \phi_{n}\right\} \subseteq K \text { iff } \phi_{1} \wedge \ldots \wedge \phi_{n} \in K
$$

Call two sentences variants iff they are accepted in exactly the same epistemic states. We are interested only in $\wedge$-variants. From $(\mathrm{CCl})$, we can infer that $\wedge$ satisfies associativity, commutativity, $\wedge$-contraction and $\wedge$-expansion, which allows us to delete parentheses. Conjunctions show a set-like behaviour which we call the variability of conjunctions. Our second assumption says that $\wedge$-variants as inputs lead to identical belief states:

$$
\text { If } \phi \text { and } \psi \text { are } \wedge \text {-variants, then } K \dot{-} \phi=K \dot{-} \psi
$$

The next principle we are going to invoke connects rational contractions with an underlying relation of epistemic entrenchment.

$$
\phi<\psi \text { iff } \psi \in K \dot{-}\langle\{\phi, \psi\}\rangle
$$

This is to express the following idea: $\psi$ is more firmly entrenched in $K$ than $\phi$ iff the agent would keep $\psi$ when facing with the need to give up (at least) one of $\phi$ and $\psi$.

Our last two principles concern the contractions which are referred to in $(\operatorname{Def}<)$. In pick contractions the goal is to retract at least one of a set of sentences from a given knowledge base $K$, i.e., to see to it that $S \nsubseteq C n(K \dot{-}\langle S\rangle)$. We know from (CCl) that in order to retract one element of $\left\{\phi_{1}, \ldots, \phi_{n}\right\}$, it is necessary and sufficient to give up $\phi_{1} \wedge \ldots \wedge \phi_{n}$. Intuitively, the latter task seems even identical with the former:

$$
K \dot{-}\left\langle\phi_{1}, \ldots, \phi_{n}\right\rangle=K \dot{-}\left(\phi_{1} \wedge \ldots \wedge \phi_{n}\right)
$$

Finally, pick contractions present by their very nature problems of rational choice. For any non-empty set of sentences $S$, the agent must make a decision which sentences of $S$ to give up in $K \dot{-}\langle S\rangle$. He must retract at least one element of $S$, but may in the case of ties of course retract several of them at the same 
time. In order to get these restrictions started, we presuppose that $S \nsubseteq C n(\emptyset)$ ). We now introduce the device of a choice function $\gamma$ such that for every $\phi \in S$,

$$
\phi \in \gamma(S) \text { iff } \phi \notin K \dot{-\langle S\rangle}
$$

In the theory of rational choice, $S$ is called the issue or the menu, and $\gamma(S)$ is called the choice set of $S$ (with respect to $\gamma$ ). As usual for choice functions, it is required here that $\emptyset \neq \gamma(S) \subseteq S$. Intuitively, $\gamma$ picks the elements of $S$ which are best to withdraw from $K$, or least secure in $S$. If both $\phi$ and $\psi$, say, are in $\gamma(S)$, this means that the agent suspends judgment on both $\phi$ and $\psi$ because no decision can be reached as to which of them is worse. We restrict ourselves to the case of finite $S$ 's in order to ensure that $(\operatorname{Def}\langle\dot{-}\rangle)$ is applicable.

The connections established by our basic principles are summarized in Fig. 1 . It is only the structure imposed on pick contractions by the vertical part of the scheme which will enable us to get an efficient translation process in the horizontal part.

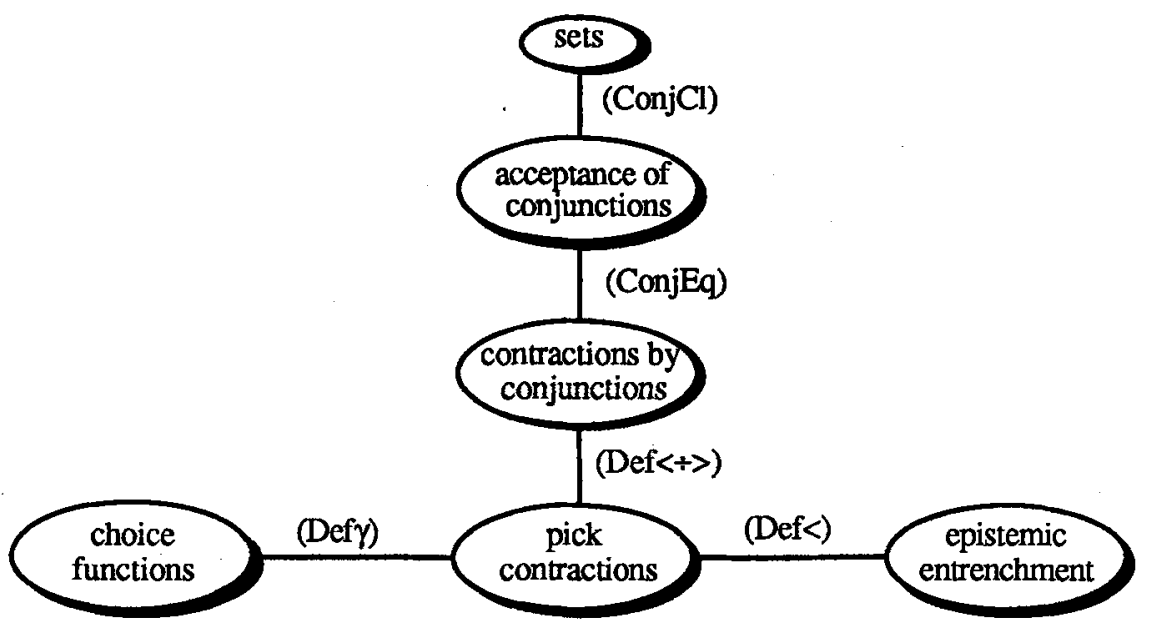

Fig. 1. Connecting choice and entrenchunent

\section{Translations}

The general argument to be used in our translations is this:

$$
\begin{aligned}
& \phi_{1} \wedge \ldots \wedge \phi_{n}<\psi_{1} \wedge \ldots \wedge \psi_{m} \\
& \text { iff } \psi_{1} \wedge \ldots \wedge \psi_{m} \in K \dot{-}\left\langle\phi_{1} \wedge \ldots \wedge \phi_{n}, \psi_{1} \wedge \ldots \wedge \psi_{m}\right\rangle \quad \text { by }(\mathrm{Def}<) \\
& \text { iff } \psi_{1} \wedge \ldots \wedge \psi_{m} \in K \dot{-}\left(\left(\phi_{1} \wedge \ldots \wedge \phi_{n}\right) \wedge\left(\psi_{1} \wedge \ldots \wedge \psi_{m}\right)\right) \text { by }(\operatorname{Def}\langle\dot{-})) \\
& \text { iff } \forall j \leq m: \psi_{j} \in K \dot{-}\left(\phi_{1} \wedge \ldots \wedge \phi_{n} \wedge \psi_{1} \wedge \ldots \wedge \psi_{m}\right) \quad \text { by (CCl), (CEq) } \\
& \text { iff } \forall j \leq m: \psi_{j} \in K \dot{-}\left\langle\left\{\phi_{1}, \ldots, \phi_{n}, \psi_{1}, \ldots, \psi_{m}\right\}\right\rangle \quad \text { by }(\operatorname{Def}\langle\dot{-}\rangle) \\
& \text { iff } \forall j \leq m: \psi_{j} \notin \gamma\left(\left\{\phi_{1}, \ldots, \phi_{n}, \psi_{1}, \ldots, \psi_{m}\right\}\right) \quad \text { by (Def } \gamma \text { ) }
\end{aligned}
$$


In short, we have for any two finite sets of sentences $S$ and $S^{\prime}$ with $S-S^{\prime} \neq \emptyset$ that $\bigwedge S<\bigwedge S^{\prime}$ iff $S^{\prime} \cap \gamma\left(S \cup S^{\prime}\right)=\emptyset$.

\subsection{From Entrenchments to Choices}

The translation process is described by the above argument read from the top to the bottom. It is actually ambiguous, due to the possibility of leaving some conjunctions unbroken. We insist, however, that the standard translation must break up every conjunction into its "atomic" parts. But we are not dealing with real atoms here. For the translation of axiom schemata below, we must keep in mind that all sentential metavariables may be instantiated by conjunctions. Hence $\phi \wedge \psi<\chi$, say, must not be converted simply into $\chi \notin \gamma(\{\phi, \psi, \chi\})$, but instead into the more general $S^{\prime \prime} \cap \gamma\left(S \cup S^{\prime} \cup S^{\prime \prime}\right)=\emptyset$, where $\phi, \psi$ and $\chi$ are thought of as the conjunctions of the elements of $S, S^{\prime}$ and $S^{\prime \prime}$, respectively.

\subsection{From Choices to Entrenchments}

The translation process is described by the above argument read from the bottom to the top. Due to the variability of conjunctions, however, the translation process is essentially ambiguous. The fully general formulation is a little complicated. Let $S_{1}^{\prime} \cup \ldots \cup S_{k}^{\prime}=S^{\prime}$ and $S_{i}$ be such that $S_{i} \cup S_{i}^{\prime}=S \cup S^{\prime}$, for every $i \leq k$. Then $S^{\prime} \cap \gamma\left(S \cup S^{\prime}\right)=\emptyset$ iff for every $i, \bigwedge S_{i}<\wedge S_{i}^{\prime}$.

\subsection{Admissible Relations}

Which relations < over the set of all sentences in our language can possibly qualify as entrenchment relations? Our basic principles limit the field of candidate relations considerably. First, we get the condition of substitutivity of variants:

If $\phi$ and $\psi$ are $\wedge$-variants, then $\phi<\chi$ iff $\psi<\chi$, and $\chi<\phi$ iff $\chi<\psi$

When considering some $\gamma(S)$ we presuppose that $S$ is finite and $S \nsubseteq C n(\emptyset)$. Recall that the concept of a choice function requires that $\gamma(S)$ be a non-empty subset of $S$. By the above translation, this means that

$$
\phi_{1} \wedge \ldots \wedge \phi_{n} \nless \phi_{i}
$$

The ambiguity in the translation of conditions of epistemic entrenchment into choice conditions is irrelevant for our concerns. On the other hand, the ambiguity involved in the converse direction is important. This is because entrenchment relations take account of the internal structure of the sentences related. We are thus presented essentially with a many-one translation. Many conditions on the side of epistemic entrenchment correspond to exactly one condition on the side of rational choice. For this reason some nontrivial necessary conditions on 
entrenchment relations are to be imposed. Our aim is to make the entrenchment relation < consistently translatable into (and a consistent translation of) a choice function $\gamma$. The key idea is the following condition which embodies in a nutshell the connection between entrenchment and choice:

$$
\phi<\psi \wedge \chi \text { iff } \phi \wedge \psi<\chi \text { and } \phi \wedge \chi<\psi
$$

Clearly, (E\&C) is a necessary condition for translatable relations $<$, because both the left-hand side and the right-hand side of (E\&C) translate into (and are translations of) $\{\psi, \chi\} \cap \gamma(\{\phi, \psi, \chi\})=\emptyset$. Now let $S_{1}^{\prime} \cup \ldots \cup S_{k}^{\prime}=S^{\prime}$ and $S_{i}$ such that $S_{i} \cup S_{i}^{\prime}=S \cup S^{\prime}$, for every $i \leq k$. It can be verified that

$$
\bigwedge s<\bigwedge S^{\prime} \text { iff } \bigwedge S_{i}<\bigwedge S_{i}^{\prime} \text { for every } i
$$

The right-hand side is the fully general translation for $S^{\prime} \cap \gamma\left(S \cup S^{\prime}\right)=\emptyset$. Our standard translation and (E\&C) suffice for deriving it.

Given (SV) and (E\&C), (NE) is equivalent to the asymmetry of $<$. We call an arbitrary relation < between sentences admissible (for the representation of entrenchments) if and only if it is asymmetric and satisfies (SV) and (E\&C). Only admissible relations can qualify as relations of epistemic entrenchment.

\section{Postulates}

\subsection{Postulates for Coherent Choice}

The constraint $(\mathrm{E} \& \mathrm{C})$ was placed on a relation $<$ since there are different ways of encoding the choices determined by $\gamma$ in one and the same issue $S$. More constraints will be imposed on entrenchment relations when coherence conditions connecting choices in different issues (of different cardinality) are respected. The following discussion is based on a set of postulates for choice functions which are well-known in the literature on rational choice and which play - with one slight modification-a central role in the model theory of belief revision in [23].

$$
\begin{gathered}
\text { If } S \subseteq S^{\prime}, \text { then } S \cap \gamma\left(S^{\prime}\right) \subseteq \gamma(S) \\
\bigcap_{i \leq k} \gamma\left(S_{i}\right) \subseteq \gamma\left(\bigcup_{i \leq k} S_{i}\right) \\
\text { If } S \subseteq S^{\prime} \text { and } \gamma\left(S^{\prime}\right) \subseteq S \text {, then } \gamma(S) \subseteq \gamma\left(S^{\prime}\right) \\
\text { If } S \subseteq S^{\prime} \text { and } \gamma\left(S^{\prime}\right) \cap S \neq \emptyset \text {, then } \gamma(S) \subseteq \gamma\left(S^{\prime}\right)
\end{gathered}
$$

Condition (I) is Sen's Property $\alpha$ and sometimes called "Chernoff"; (II) is a finite version of Sen's Property $\gamma$, also called "expansion axiom"; (III) is sometimes called "Aizerman's axiom"; (IV) is Sen's Property $\beta+$, also known as "Dual Chernoff". Notice that (IV) implies both (II) and (III). As there are many relations < satisfying (I) - (IV), this set of postulates is consistent. 
A relation $<$ is called modular iff $\phi<\psi$ entails that either $\phi<\chi$ or $\chi<\psi$, for every $\chi$. Modularity and asymmetry jointly entail transitivity. A choice function $\gamma$ is called (transitively, modularly) rationalizable iff there is a (transitive, modular) preference relation < such that for all $S$,

$$
\gamma(S)=\{\phi \in S: \text { there is no } \psi \in S \text { such that } \psi<\phi\}
$$

The following facts have been common knowledge in the theory of rational choice at least since Sen [25].

Observation 1 Let $\gamma$ be a choice function which can take all finite subsets of a given domain as arguments.

(a) $\gamma$ is rationalizable iff it is rationalizable by the preference relation defined by

$$
\phi<\psi \text { iff } \psi \notin \gamma(\{\phi, \psi\})
$$

(b) $\gamma$ is rationalizable iff it satisfies (I) and (II);

(c) $\gamma$ is transitively rationalizable iff it satisfies (I), (II), and (III);

(d) $\gamma$ is modularly rationalizable iff it satisfies (I) and (IV).

Condition (*) is a typical definition of "revealed preferences" which are widely used in the theory of choice and preference. It is the asymmetric version of the "base preferences" originating with H. Uzawa and K. Arrow. Condition (*) above can be obtained by conjoining (Def $<$ ) with (Def $\gamma$ ). Epistemic entrenchment is just a certain kind of revealed preference. It is more general insofar as it can take combinations (i.e., conjunctions) of elements rather than only single elements as arguments. The objects between which to choose possess an internal structure.

\subsection{Postulates for Epistemic Entrenchment}

Essentially the following set of postulates is advocated as a set of conditions suitable for capturing the notion of epistemic entrenchment in Rott [21], where a corresponding set of postulates for belief revision is specified.

$$
\begin{gathered}
\phi \nless \phi \\
\text { If } \phi<\psi \wedge \chi, \text { then } \phi<\psi \\
\text { If } \phi<\psi, \text { then } \phi \wedge \chi<\psi \\
\text { If } \phi<\psi \text { and } \phi<\chi, \text { then } \phi<\psi \wedge \chi \\
\text { If } \phi \wedge \psi<\psi, \text { then } \phi<\psi
\end{gathered}
$$

If we add the postulate of virtual connectivity or modularity,

$$
\text { If } \phi<\psi \text {, then } \phi<\chi \text { or } \chi<\psi
$$

we get essentially the concept of epistemic entrenchment as originally introduced by Gärdenfors and Makinson [10]. We refer to relations satisfying (EE1) - $\left(\mathrm{EE} 3^{\downarrow}\right)$ 
as generalized relations of epistemic entrenchment, and to those satisfying in addition (EE4) as standard relations of epistemic entrenchment. Modularity is a very powerful condition. It corresponds to the condition of Rational Monotonypostulate $(-8)$ in the AGM nomenclature--in the theory of belief revision. For the theory of epistemic entrenchment, it has the following consequences:

Observation 2 Suppose the relation < satisfies (EE1) and (EE4). Then (a) $<$ satisfies (EE2 ${ }^{\uparrow}$ ) iff it satisfies (EE2 $\downarrow$ ); (b) if < satisfies (EE3 ${ }^{\uparrow}$ ), then it satisfies $\left(E E 3^{\downarrow}\right)$; if $<$ is asymmetric and satisfies (EE3 $\downarrow$ ), then it also satisfies $\left(E E 3^{\uparrow}\right)$.

Relations of epistemic entrenchment with comparability can thus be characterized, e.g., by postulates (EE1), (EE2 $\left.{ }^{\uparrow}\right),\left(\mathrm{EE} 3^{\uparrow}\right)$ and (EE4). There are also minimality and maximality conditions in [10] which correspond to the following constraints on $\gamma$ :

$$
\begin{array}{cr}
\gamma(S)=S-K \text { iff } S-K \neq \emptyset & \text { (Minimality) } \\
\gamma(S) \cap C n(\emptyset)=\emptyset, \text { unless } S \subseteq C n(\emptyset) & \text { (Maximality) }
\end{array}
$$

These conditions relate $\gamma$ to $K$ and $C n$ and are thus not purely structural conditions of rational choice.

\section{The Postulates Translated}

\subsection{From Coherent Choice to Epistemic Entrenchment}

We wish to see whether some widely accepted coherence criteria for choices are already taken account of by the usual requirements for epistemic entrenchment relations. By (non-trivial) application of the translation specified in Section 3.2, we get the following constraints on entrenchment relations.

$$
\begin{gathered}
\text { If } \phi<\psi, \text { then } \phi \wedge \chi<\psi \\
\text { If } \phi \wedge \psi<\chi, \text { then } \phi<\chi \text { or } \psi<\chi \\
\text { If } \phi \wedge \psi<\chi \text { and } \phi \wedge \chi<\psi, \text { then } \phi<\chi \\
\text { If } \phi \wedge \psi<\chi \text { and } \psi \nless \phi \wedge \chi, \text { then } \phi<\chi
\end{gathered}
$$

Relations satisfying - at least some interesting selection from-the postulates (EI) - (EIV) can be taken to exhibit features of rational choice.

\subsection{From Epistemic Entrenchment to Coherent Choice}

By (straightforward) application of the translation specified in Section 3.1 , we get the following constraints on choice functions.

$$
S \cap \gamma(S) \neq \emptyset
$$




$$
\begin{gathered}
\text { If }\left(S \cup S^{\prime}\right) \cap \gamma\left(S \cup S^{\prime} \cup S^{\prime \prime}\right)=\emptyset \text {, then } S^{\prime} \cap \gamma\left(S^{\prime} \cup S^{\prime \prime}\right)=\emptyset \\
\text { If } S \cap \gamma\left(S \cup S^{\prime}\right)=\emptyset \text {, then } S \cap \gamma\left(S \cup S^{\prime} \cup S^{\prime \prime}\right)=\emptyset \\
\text { If } S^{\prime} \cap \gamma\left(S \cup S^{\prime}\right)=\emptyset \text { and } S^{\prime \prime} \cap \gamma\left(S \cup S^{\prime \prime}\right)=\emptyset, \\
\text { then }\left(S^{\prime} \cup S^{\prime \prime}\right) \cap \gamma\left(S \cup S^{\prime} \cup S^{\prime \prime}\right)=\emptyset \\
\text { If } S \cap \gamma\left(S \cup S \cup S^{\prime}\right)=\emptyset, \text { then } S \cap \gamma\left(S \cup S^{\prime}\right)=\emptyset \\
\text { If } S \cap \gamma\left(S \cup S^{\prime}\right)=\emptyset, \text { then } S \cap \gamma\left(S \cup S^{\prime \prime}\right)=\emptyset \\
\text { or } S^{\prime \prime} \cap \gamma\left(S^{\prime} \cup S^{\prime \prime}\right)=\emptyset
\end{gathered}
$$

\section{Implications}

We now study the relative strength of the postulates for choice and entrenchment.

\subsection{Implications between Choice Postulates}

Observation 3 (a) (CC1) holds for every choice function $\gamma$; (b) (CC2 ${ }^{\uparrow}$ ) is

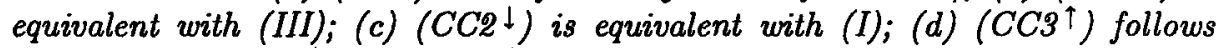

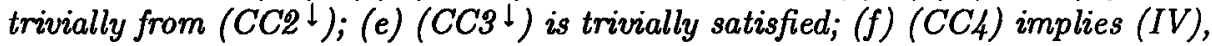
and follows from the conjunction of (I) and (IV).

Part (d) of Observation 3 shows that although (EE3 ${ }^{\uparrow}$ ) is logically independent from (EE2 $\left.{ }^{\downarrow}\right)$ in the theory of epistemic entrenchment, the translations are not. Part (e) tells us that although (EE3 ${ }^{\downarrow}$ ) in non-trivial, its translation is. The explanation for these surprising facts is that given the admissibility of $<,\left(\mathrm{EE} 3^{\uparrow}\right)$ is just a weakening of (EE2 $\downarrow$ ), and (EE3 $\downarrow$ ) does not add anything new. Together with Observation 1, parts (c) and (f) of Observation 3 reveal a new axiomatization of modularly rationalizable choice functions: A choice function is rationalizable by a modular preference relation iff it satisfies $\left(\mathrm{CC} 2^{\downarrow}\right)$ and $(\mathrm{CC} 4)$.

\subsection{Implications between Entrenchment Postulates}

We know from the previous section and the translatability of choice conditions into conditions for entrenchment relations that certain equivalences must hold between the original conditions set up in [10] and [21] and the ones derived from coherent choice. Still it is instructive to study in detail the interrelations at the level of entrenchment relations.

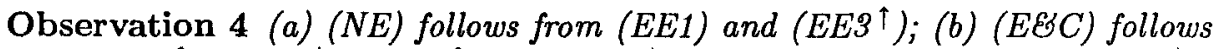
from (EE2 $\left.{ }^{\uparrow}\right),\left(E E 2^{\downarrow}\right),\left(E E 3^{\uparrow}\right)$ and $\left(E E 3^{\downarrow}\right) ;(c)$ (EI) is identical with (EE2 $\left.{ }^{\downarrow}\right)$; (d) (EII) follows from (EE1), (EE3 ${ }^{\uparrow}$ ) and (EE4); (e) (EIII) follows from (EE2 ${ }^{\uparrow}$ ), $(E E 2 \downarrow),\left(E E 3^{\uparrow}\right)$ and $\left(E E 3^{\downarrow}\right) ;(f)(E I V)$ follows from (EE2 $\left.\downarrow\right),\left(E E 3^{\downarrow}\right)$ and (EE4). 
Observation 5 (a) (EE1) follows from (NE); (b) (EE2 $\uparrow$ ) follows from (EBC) and (EIII); (c) (EE2 ${ }^{\downarrow}$ ) is identical with (EI); (d) (EE3 ${ }^{\uparrow}$ ) follows from (EEC) and (EI); (e) (EE3 ل) follows from (EIII); $(f)$ (EE4) follows from (EBC), (EI), (EIII) and (EIV).

For admissible relations <, (EIV) implies both (EII) and (EIII), just as (IV) implies (II) and (III). Thus asymmetry, (SV), (E\&C), (EI) and (EIV) provide an alternative axiomatization of epistemic entrenchment.

\section{Conclusion and Related Work}

Let us summarize the major results of the previous sections in non-technical terms, thereby answering the questions asked in the introduction:

- The entrenchment postulates (EE1), (EE2 $\left.{ }^{\downarrow}\right),\left(\mathrm{EE}^{\dagger}{ }^{\uparrow}\right)$ and $\left(\mathrm{EE} 3^{\downarrow}\right)$, translated into constraints on the choice of retractible sentences, are satisfied by all rationalizable choice functions. In addition, $\left(\mathrm{EE} 2^{\uparrow}\right)$ is satisfied by all transitively rationalizable choice functions, and (EE4) is satisfied by all modularly rationalizable choice functions.

- The choice postulates (I) and (III), translated into constraints on the entrenchment of sentences, as well as the general admissibility constraints imposed by the interpretation of entrenchment in terms of choices, are satisfied by all generalized relations of epistemic entrenchment. In addition, the choice postulates (II) and (IV) are satisfied by all standard relations of epistemic entrenchment.

- All admissible relations < satisfying the choice postulates (I) and (III), translated into constraints on the entrenchment of sentences, are generalized relations of epistemic entrenchment. If < in addition satisfies (IV), translated into a constraints on the entrenchment of sentences, it is a standard relations of epistemic entrenchment.

In a companion paper [24], we study the correspondences between choice postulates and systems of nonmonotonic reasoning which are based on choices or preferences between default assumptions or expectations. It turns out that (I) corresponds to the condition "Or", (II) to a weakened form of Disjunctive Monotony, (III) to Cumulative Monotony, and (IV) to Rational Monotony. (For all these terms, see $[6,13,16,17]$.) It is interesting that seen from the present perspective, Disjunctive Monotony is at least as plausible as Cumulative Monotony.

The focus of the present paper has been the concept of epistemic entrenchment (or that of an "expectation ordering" [11]). However, we gave no material explanation where the entrenchment relations might come from. What we have attempted to give is rather a theoretical explanation or justification of the logical constraints for such relations. We now briefly discuss alternative ways of doing this. 
First of all, we note that in the literature one can distinguish qualitative-relational from quantitative-numerical approaches, with the obvious connection that $\phi<\psi$ (where ' $<$ ' is epistemic entrenchment) iff $\operatorname{Ent}(\phi)<\operatorname{Ent}(\psi)$ (where ' $<$ ' is the usual less-than relation over ordinal or real numbers). More importantly, we can also distinguish comparative-necessity based approaches from comparativepossibility based approaches, with the connection $\phi<\psi$ iff $\neg \phi \prec \neg \psi$, or $\operatorname{Ent}(\phi)=\operatorname{Plaus}(\neg \phi)$ (see, e.g., [11, Appendix A2]). Here $\neg \phi \prec \neg \psi$ is to be understood as saying that $\neg \phi$ is closer to one's present beliefs, or more plausible, than $\neg \psi$, and similarly, smaller Plaus-values signify a higher plausibility. Epistemic entrenchment is perhaps the best known example of the former kind, while various people are working in the second framework, for instance, Lewis, Spohn, Grove, and Freund. In much of the work of Dubois and Prade the connection between comparative necessity and possibility is carefully discussed.

There have been several attempts to give a natural derivation of relations of comparative necessity or possibility. (In the following, we mean by an ordering a weak ordering in which ties are permitted.) Firstly, one can derive an ordering of propositions from an ordering of models. The basic idea is best expressed in terms of comparative possibility and says that $\operatorname{Plaus}(\phi)=\inf \{\operatorname{Plaus}(\omega)$ : $\omega$ satisfies $\phi\}$. The usual logical constraints on comparative possibility are then automatically satisfied. This idea can be traced back at least to David Lewis' [14] construction of comparative possibilities out of comparative similarities and Lofti A. Zadeh's [27] construction of a possibility measure out of a possibility distribution. The latter approach has been turned into a powerful research program by Dubois and Prade and their collaborators (see, e.g., $[2,5])$. The former approach has been extended and modified by Grove [12] and Spohn [26], and also- as we now know with the benefit of hindsight--in the research paradigm of Alchourrón, Gärdenfors and Makinson $[1,8,11,19]$. Here the necessity-like concept of epistemic entrenchment has been constructed from a preference relation of maximal non-implying subsets (which in turn stand in a 1-1-correspondence to the non-models) of the current belief set. A more general model-theoretic characterization of comparative possibility without connectivity has been given by Freund [6]. Except for a limiting case condition for logical truths, his postulates for "preferential preorderings" are exact duals of the postulates for generalized epistemic entrenchment of Rott [21].

Secondly, one can draw on syntactical constructions starting from arbitrary (partial) orderings of the sentences in a belief base. Here one can distinguish a positive and a negative idea. The first one, suggested for the first time by Dubois and Prade [4], judges the entrenchment of $\phi$ according to the weakest elements in any proof set for $\phi$. A more general version of this idea without connectivity assumptions has independently been used in [20]. The second one, suggested in Rott [22], judges the entrenchment of $\phi$ according to the most conservative or economical way to discard $\phi$. It has been shown that in the context of prioritized belief bases this relation is identical with the relation studied in the present paper (minimal change vs. competitive interpretation of epistemic entrenchment [22]), 
and that it can be used to approximate prioritized base contractions in the style of Nebel [18]. It is not difficult to show that the negative idea leads to a (proper) extension of epistemic entrenchment relation < obtained from formalizing the positive idea. However, the usual way of applying epistemic entrenchment relations $[10,21]$ does not lead to very good results in the context of belief bases (as opposed to belief sets which are closed under $C n$ ), since they suffer from what Benferhat et al. [2] call the "drowning effect".

Thirdly, entrenchment relations have been reconstructed from contraction behaviour over full belief sets (Gärdenfors and Makinson [10, 21]). This is evidently very close the the approach followed in this paper, but the crucial and strong AGM postulates $(K \dot{-} 7)$ and $(K \dot{-})$ are not as systematically motivated as the criteria studied in rational choice theory.

Finally, we mention that the decision-theoretic or choice-theoretic framework has already been systematically employed in belief revision and nonmonotonic reasoning by Lindström [15] and Rott [23]. There, however, preferences and choices are considered on the model-theoretical level, and no relations between sentences are considered. Correspondingly, problems of conceptualization, postulates for contractions, and methods of proof are quite different. Perhaps the first author to point out the relevance of the theory of choice and its economic sense of rationality for belief revision was Doyle [3], who however used preferences over sets of sentences and did not study the implications of criteria of rational choice for the logic of belief change.

\section{References}

1. Alchourrón, Carlos, Peter Gärdenfors and David Makinson: 1985, "On the logic of theory change: Partial meet contraction functions and their associated revision functions", Journal of Symbolic Logic 50, 510-530.

2. Benferhat, Salem, Claude Cayrol, Didier Dubois, Jérôme Lang and Henri Prade: 1993, "Inconsistency management and prioritized syntax-based entailment", in R. Bajcsy (ed.), Proceedings of the 13th International Joint Conference on Artificial Intelligence, Morgan Kaufmann, San Mateo, Ca, 640-645.

3. Doyle, Jon: 1991, "Rational belief revision: Preliminary report", in J. Allen, R. Fikes and E. Sandewall (eds.), Principles of Knowledge Representation and Reasoning: Proceedings of the 2nd International Conference, Morgan Kaufmann, San Mateo, Ca., 163-174.

4. Dubois, Didier, and Henri Prade: 1987 , "Necessity measures and the resolution principle", IEEE Transactions on Systems, Man and Cybernetics 17, 474-478.

5. Dubois, Didier, and Henri Prade: 1991, "Epistemic entrenchment and possibilistic logic", Artificial Intelligence 50, 223-239.

6. Freund, Michael: 1993, "Injective models and disjunctive relations", Journal of Logic Programming 3, 231-247.

7. Gärdenfors, Peter: 1984, "Epistemic importance and minimal changes of belief", Australasian Journal of Philosophy 62, 136-157. 
8. Gärdenfors, Peter: 1988, Knowledge in Flux: Modeling the Dynamics of Epistemic States, Bradford Books, MIT Press, Cambridge, Mass.

9. Gärdenfors, Peter (ed.): 1992, Belief Revision, Cambridge University Press, Cambridge.

10. Gärdenfors, Peter, and David Makinson: 1988, "Revisions of knowledge systems using epistemic entrenchment", in M. Vardi (ed.), Theoretical Aspects of Reasoning About Knowledge, Morgan Kaufmann, Los Altos, pp. 83-95.

11. Gärdenfors, Peter, and David Makinson: 1994, "Nonmonotonic Inference Based on Expectations", Artificial Intelligence 65, 197-245.

12. Grove, Adam: 1988, "Two modellings for theory change", Journal of Philosophical Logic 17, 157-170.

13. Lehmann, Daniel, and Menachem Magidor: 1992, "What does a conditional knowledge base entail?", Artificial Intelligence 55, 1-60.

14. Lewis, David: 1973, Counterfactuals, Blackwell, Oxford.

15. Lindström, Sten: 1991, "A semantic approach to nonmonotonic reasoning: Inference operations and choice", Department of Philosophy, University of Uppsala, Uppsala Prints and Preprints in Philosophy, Number 6.

16. Makinson, David: 1990 , "General patterns in nonmonotonic reasoning", in D.M. Gabbay a.o. (eds.), Handbook of Logic in Artificial Intelligence and Logic Programming, Vol. II: Nonmonotonic and Uncertain Reasoning, Oxford UP, Oxford, to appear.

17. Makinson, David, and Peter Gärdenfors: 1991, "Relations between the logic of theory change and nonmonotonic logic", in A. Fuhrmann and M. Morreau (eds.), The Logic of Theory Change, Springer LNCS 465, Berlin etc., pp. 185-205.

18. Nebel, Bernhard: 1992, "Syntax-based approaches to belief revision", in [9], pp. $52-88$.

19. Rott, Hans: 1991, "Two methods of constructing contractions and revisions of knowledge systems", Journal of Philosophical Logic 20, 149-173.

20. Rott, Hans: 1992, "On the logic of theory change: More maps between different kinds of contraction function", in [9], pp. 122-141.

21. Rott, Hans: 1992, "Preferential belief change using generalized epistemic entrenchment", Journal of Logic, Language and Information 1, 45-78.

22. Rott, Hans: 1992, "Modellings for belief change: Prioritization and entrenchment", Theoria 58, to appear.

23. Rott, Hans: 1993, "Belief contraction in the context of the general theory of rational choice", Journal of Symbolic Logic 58, 1426-1450.

24. Rott, Hans: 1994, "Nonmonotonic logic = monotonic logic + choice", Manuscript, University of Konstanz, June 1994.

25. Sen, Amartya K.: 1971, "Choice functions and revealed preference", Review of Economic Studies 38, 307-317.

26. Spohn, Wolfgang: 1988, "Ordinal Conditional Functions", in William L. Harper and Brian Skyrms (eds.), Causation in Decision, Belief Change, and Statistics, vol. II, Reidel, Dordrecht, 105-134.

27. Zadeh, Lofti A.: 1978, "Fuzzy sets as a basis for a theory of possibility", Fuzzy Sets and Systems 1, 3-28. 